\title{
Documents pour la connaissance de la tératologie des Trématodes \\ Anomalies chez Brachylaemus erinacei E. Blanchard 1847
}

Nous avons eu l'occasion de disséquer, le 5 novembre 1966, un Hérisson du sexe femelle, capturé aux environs d'Aubagne (1) (B.-du-Rh.), qui était abondamment parasité par un Distome que nous rapportons à l'espèce classique Brachylaemus erinacei E. Blanchard, 1847, (forme non spinulée). Nous avons recueilli 46 individus, localisés dans les 15 derniers centi-
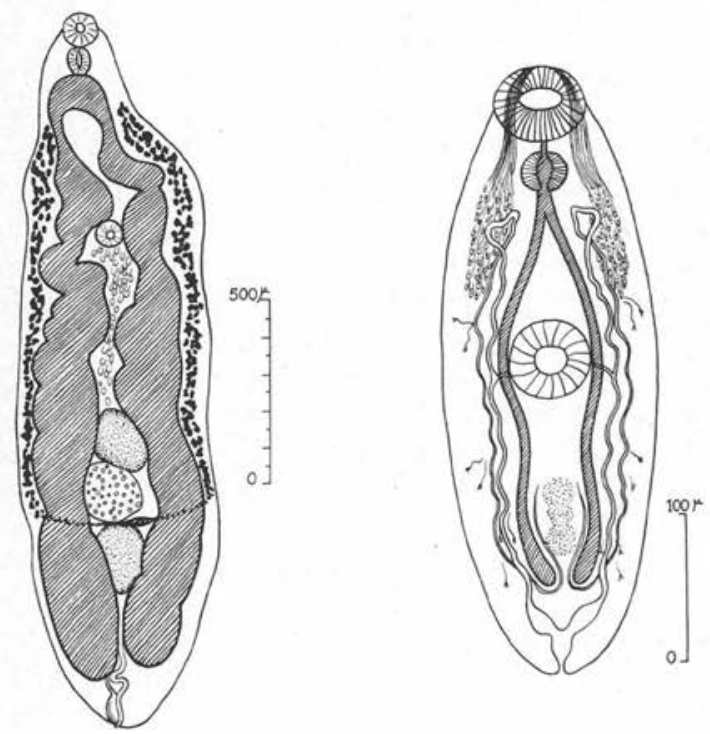

FIG. 1. - Brachylaemus erinacei E. Blanchard. Sujet normal avec ovaire en position intertesticulaire

mètres de l'intestin; ils étaient en pleine nutrition: la plupart avaient les cæcums gorgés de sang. Sur ces 44 sujets, deux ont attiré notre attention car ils présentaient des anomalies que nous figurons ici (fig. 2 et 3 ).

On sait que, chez Brachylaemus, lovaire occupe normalement une position médiane entre les deux testicules; il est accompagné de l'ootype qui lui est accolé ; c'est donc à partir de ce point que l'utérus se dirige vers la région antérieure (fig. 1). Les vitelloductes décrivent une courbe à concavité antérieure en contournant le bord postérieur de l'ovaire et viennent aboutir au réservoir vitellin qui est situé entre l'ovaire et le testicule postérieur. Les deux branches intestinales qui s'étendent jusqu'à l'extrémité du corps s'appliquent étroitement contre le testicule postérieur.

(1) Nous exprimons nos meilleurs remerciements à M. Guy Balansard qui a eu l'amabilité de nous procurer ce matériel. 

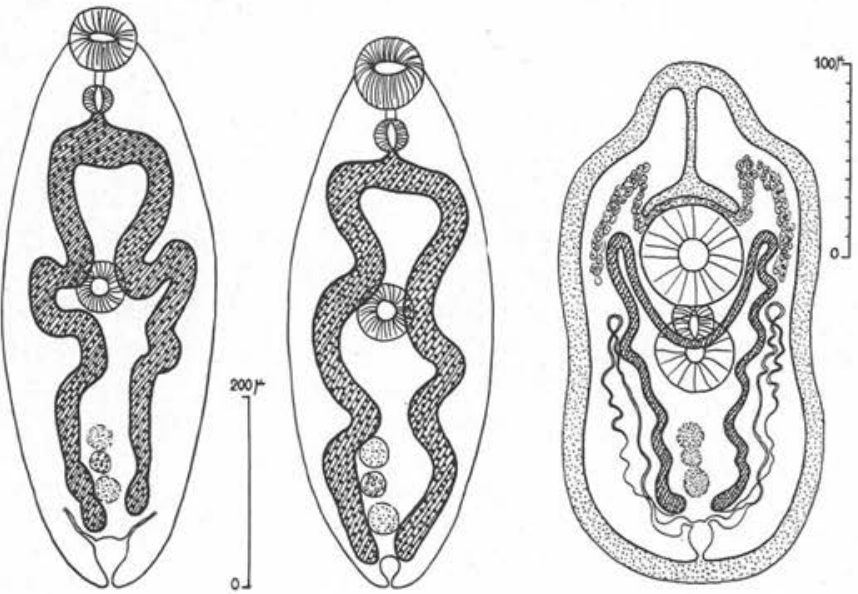

FIG. 2. - Sujet tératologique : testicules contigus; ovaire, ootype et réservoir vitellin reportés en arrière

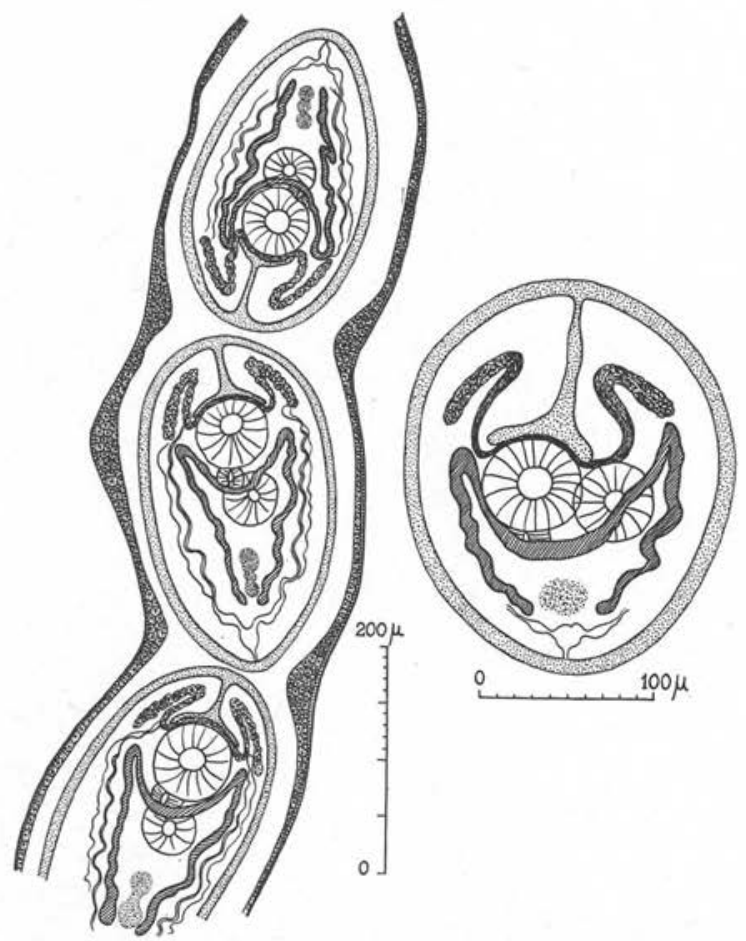

FIG. 3. - Autre sujet tératologique. Même topographie des organes. On distingue une anse utérine qui contourne le testicule postérieur. L'ootype est très apparent en arrière de l'ovaire 
Chez nos deux exemplaires tératologiques, les testicules sont accolés et l'ovaire est reporté en arrière, à l'extrémité du corps ; l'ootype l'a accompagné dans son déplacement, ce qui a eu pour effet d'allonger le trajet de l'utérus; il y a, en outre, des modifications qui concernent les vitelloductes : leur courbe contourne le testicule postérieur; le réservoir vitellin, très visible sur la photographie 3, vient se placer entre le testicule postérieur et l'ovaire.

On a observé, assez souvent chez les Distomes, des anomalies tératologiques portant soit sur l'atrophie, soit sur la duplication des gonades. C'est ainsi que F. Pick (1949) a décrit un exemplaire de Watsonius watsoni (Conyngham, 1904) avec un seul testicule; l'un de nous a décrit et figuré (1965, p. 33) un cas de monorchidie chez Prosthogonimus ovatus (Rudolphi). V. M. Alekseev et S. M. Lopukhova (1962) ont observé l'anomalie inverse chez Prosthogonimus pellucidus (v. Linstow): un sujet avec quatre testicules; les mêmes auteurs ont encore signalé la monorchidie chez Echinoparyphium recurvatum (v. Linstow). Un cas de dédoublement de l'ovaire chez Metorchis pinguinicola Skrjabin a été aussi étudié par Alekseev (1963).

L'anomalie que nous signalons ici chez Brachylaemus erinacei présente un certain intérêt parce que le déplacement des gonades entraîne une série de modifications dans les rapports anatomiques concernant l'ensemble des organes génitaux.

\section{Bibliographie}

Alekseev (V. M.), 1963. - Une modification tératologique de Metorchis pinguinicola Skrjabin (en Russe). Zool. Zhurn. XLII, (12), 1871.

— et LopuKhova (S. M.), 1962. - Tératologie des Trématodes (en Russe). Zool. Zhurn. $X L I,(3), 453-455$.

Dollfus (R.-Ph.), 1934-35. - Sur quelques Brachylaemus de la faune française récoltés principalement à Richelieu (Indre-et-Loire). Ann. Parasitol. Hum. et Comp., XII, 551-575 et XIII, 52-79.

PICK (E.), 1949. - Un cas tératologique chez le Trématode Watsonius watsoni (Conyngham, 1910). Ann. Parasitol. Hum. et Comp., XXIV, 28-29.

Trmon-David (J.), 1965. - Trématodes parasites des Odonates. Biologie et cycles. Ann. Fac. Sc. Marseille, XXXVIII, 15-41.

Jean TIMon-David et Pierre Timon-David. 